\title{
INNOVATIVE MICRO-WALKING ROBOT USING FLEXIBLE MICROACTUATOR
}

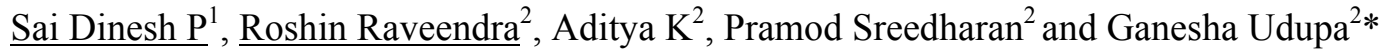 \\ ${ }^{1}$ Department of Electronics and Communication Engineering, Amrita Vishwa Vidyapeetham, Kerala, India \\ ${ }^{2}$ Department of Mechanical Engineering, Amrita Vishwa Vidyapeetham, Kerala, India \\ *Corresponding Author (ganesh@am.amrita.edu)
}

\begin{abstract}
In the area of Robotics, the micro-walking robots play a very important role as it is required to perform inspection and maintenance inside pipelines or narrow spaces where people cannot enter. Extensive research work is under way in the design and control of the micro-walking robot. An exhaustive survey of all such robots conveys the idea of higher and higher sophistication with innumerable components and elaborate controls with programmable ability. Microwalking robots proposed by earlier researchers use flexible micro actuators that have two or more internal chambers whose internal pressures are controlled independently. The proposed micro-walking robot uses flexible micro actuators (FMA) that are having single internal chamber with an innovative Electro-pneumatic control algorithm (EPCA), simple feedback mechanism and which are compact and easy to manufacture. The present work has paved the way for extensive research on this innovative technique as it holds out the true potential for very interesting applications in various areas such as robot grippers, pipeline inspection robots and underwater robots.
\end{abstract}

Keywords: Asymmetric Tube, Electro-pneumatic Control Algorithm (EPCA), Flexible Micro Actuator (FMA)

\section{INTRODUCTION}

Micro-walking robots play an important role to inspect and diagnose pipelines of nuclear power stations, chemical plants, and town gas supply and so on. Using robots for these tasks enables humans from doing dangerous works and they will be more precise.

The essential problems in realizing autonomous walking robots consist of poor actuation systems and high power consumption rate. Autonomous walking robots hence create an interesting area of research to develop and refine the application of possible actuation systems and development of flexible control capability using embedded systems. Nowadays, walking robots are emerging from laboratories to serve in a variety of non-industrial roles: as underwater explorers, as toys, as nuclear plant repair and decommissioning tools, and even as extraterrestrial explorers.

Various studies have been made for conventional size walking robots. However they usually need many devices such as electric motors, encoders, reduction gears, foot touch sensors, bearings, and mechanical links. Conventional design technique for walking robots results in robots which have complicated mechanisms and a large number of electrical cables between the robots and the controllers [1-2].

The authors have developed a flexible micro actuator (FMA) for miniature robots, which is made of nitrile rubber and is driven electro-pneumatically. Different from the existing FMA which are three chambered [3-6] that have three degrees of freedom, we have developed single chambered asymmetric FMA which has single degree of freedom. And also various studies have been made for the material of the FMA, sensors and the control algorithms.

\section{PRINCIPLE OF FLEXIBLE MICROACTUATOR}

The principle of working is exactly opposite to that of the principle on which the bourdon tube is working. An over 
simplified statement of this new principle is to dub it as an anti-bourdon tube principle [7]. The bourdon tube used is initially in curved form with flat or elliptic cross section which under the application of internal pressure will try to stretch because of the action of the flat or elliptic section becoming circular under pressure. Contrary to this a straight asymmetric (eccentric) tube with circular cross section under the application of pressure will become curved and elliptic in cross section.

\section{ILLUSTRATION OF THE PRINCIPLE}

When the wall thickness is made asymmetric, the tube under internal pressure will curve in the direction of the side having greater thickness as the expansion of the thinner side (outside radius) will be more than thicker side (inside radius) because of the unequal thickness as shown in Fig. 1 This effect is due to dual effect of an unequal expansion or in other words differential expansion of the tube at various points and the end moment induced due to asymmetry of cross section. [8].

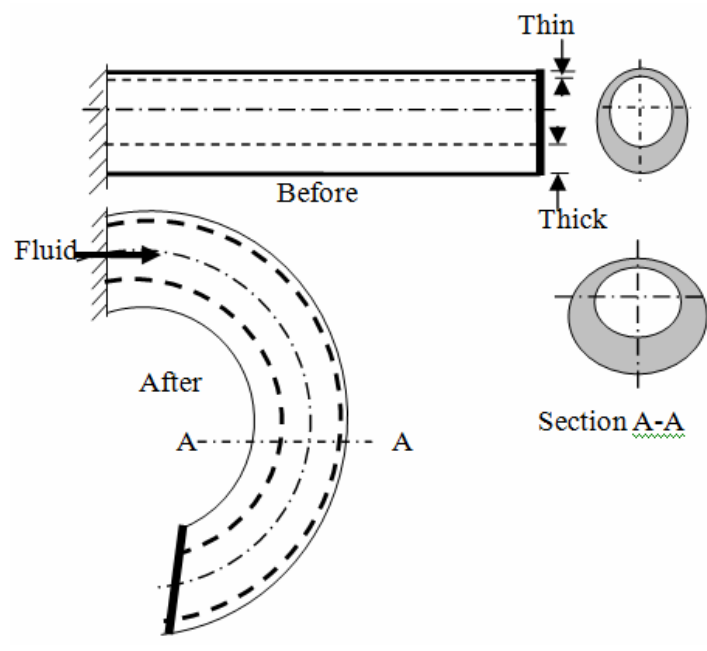

Fig. 1 Bending of asymmetric tube actuator subjected to pneumatic pressure.

To find the deflection in an asymmetric tube actuator subjected to internal pressure, the Euler equation is used.

Fig. 2 shows bending of asymmetric tube actuator subjected to pressure. When fluid pressure is applied with the free end closed, it bends due to combined effect of an end moment which develops at the free end due to eccentricity and due to differential expansion of the top and bottom fibers.

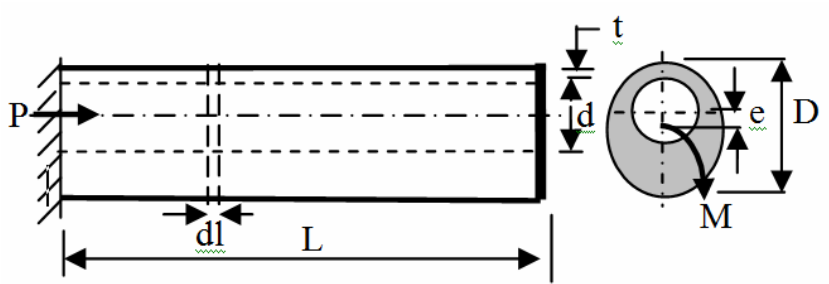

Fig. 2 Bending of asymmetric tube actuator subjected to pressure.

By Euler's Formulae [9],

$\frac{E l \frac{d^{2} y}{d x^{2}}}{\left[1+\left(\frac{d^{2} y}{d x^{2}}\right)^{2}\right]^{3 / 2}}=P A e=M$

where E is Young's modulus, e is eccentricity, $\mathrm{P}$ is Pressure applied to the FMA, I is the second moment of area.

Substituting, $\frac{d y}{\Delta x}=v$ in (1), we get

$\frac{E I v^{\prime}}{\left[1+(v)^{2}\right]^{3 / 2}}=P A e$

Let $v=\tan \theta$

where is the angle of deflection with respect to $\mathrm{x}$-axis.

By differentiating,

$d v=\left(\sec ^{2} \theta\right) d \theta$

Substituting in (2)

$E I \frac{\left(\sec ^{2} \theta\right) d \theta}{\left[\sec ^{2} \theta\right]^{3 / 2}}=$ PAedx

Integrating, we get

$$
E I \sin \theta=P A e x+K
$$

Applying the boundary condition, at $\mathrm{x}=0, \theta=0$ and hence $\mathrm{K}=0$,

$\theta=\sin ^{-1}\left(\frac{C P A E x}{E I}\right)$

where $\mathrm{C}$ is an empirical constant which compensates the dynamic effect of bending of FMA and the value of $\mathrm{C}$ is 1.48 .

Radius of curvature at any pressure is

$R=\frac{E I}{C P A \theta}$

Fig 3 shows the comparison between the theoretical and 
experimental angles of deflection with respect to the pressure. Angle of deflection increases with increase in pressure for both the theoretical and experimental values.

Fig. 4 shows the comparison between the theoretical and experimental radii of curvature with respect to the pressure.

Radius of curvature decreases with increase in pressure for both the theoretical and experimental values.

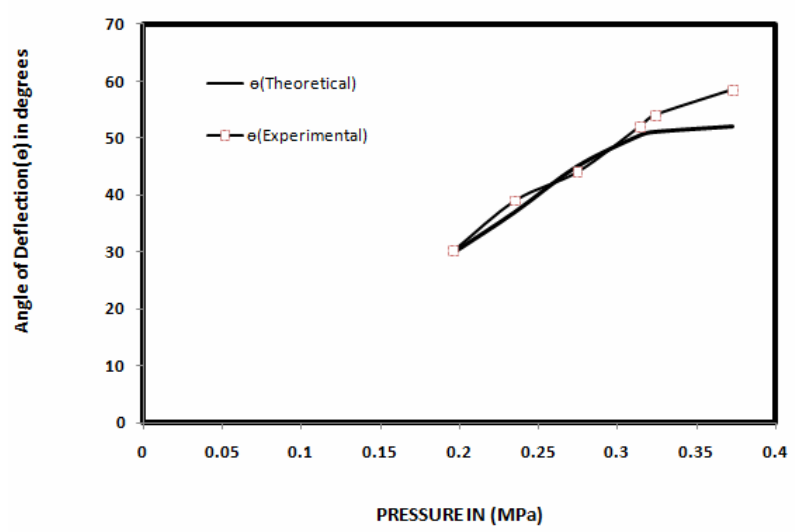

Fig. 3 Comparison of theoretical and experimental data for angle of deflection vs pressure.

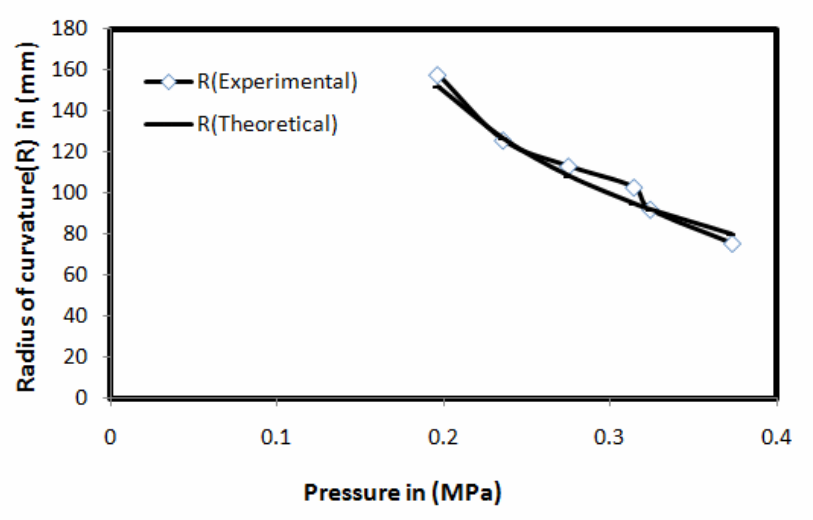

Fig. 4 Comparison of theoretical and experimental data for radius of curvature vs pressure.

Experiments were conducted using various materials such as rubber, poly-urethane, nitrile etc. The nitrile rubber tubes (synthetic materials) were found to be more effective than the natural rubber tubes. However the difficulty in obtaining the desired materials imposed some restrictions on the experiments. The behavior observed was consistent and reliable. Properly designed components would be desirable for industrial applications involving positional accuracy; reliability and stability are essential requirements for any field application. Fig. 5 shows the SolidWorks simulation of bending of an asymmetric tube (synthetic) of length $100 \mathrm{~mm}$ subjected to varying pressure. The tube is having inner diameter of $6 \mathrm{~mm}$ and outer diameter of $12 \mathrm{~mm}$ with eccentricity of $2 \mathrm{~mm}$.

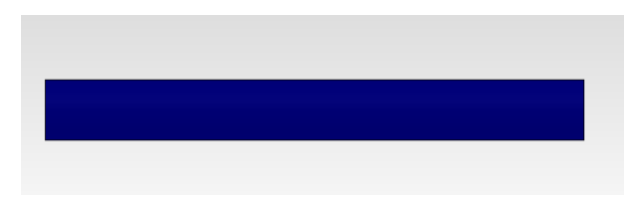

(a) $\mathbf{P}=0 \mathrm{MPa}$

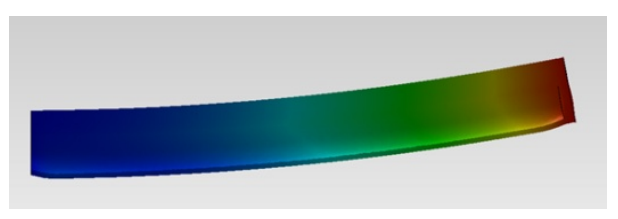

(b) $\mathbf{P}=0.2 \mathrm{MPa}$

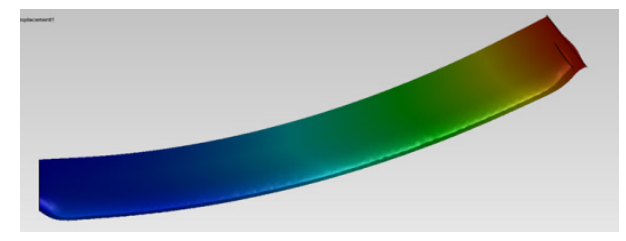

(c) $\mathrm{P}=0.35 \mathrm{MPa}$

Fig. 5 Analytical results of deformations of the actuator.

Fig. 6 shows the experimental results of deformation of the actuator at different pressures.

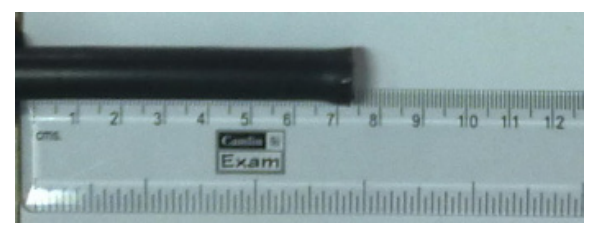

(a) $\mathbf{P}=\mathbf{0 M P a}$

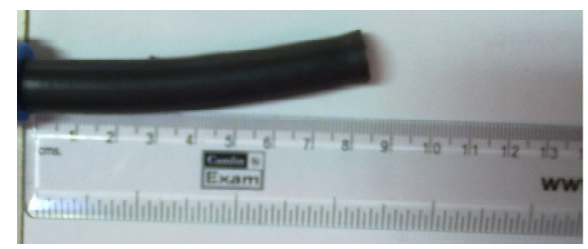

(b) $\mathrm{P}=0.2 \mathrm{MPa}$ 


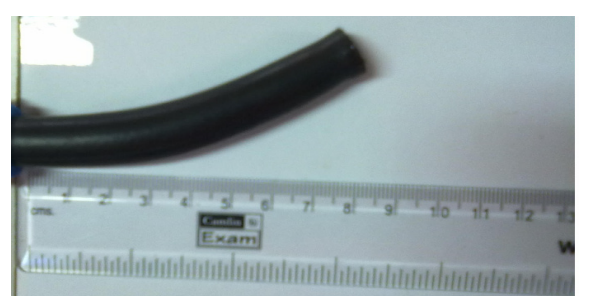

(c) $\mathrm{P}=0.35 \mathrm{MPa}$

Fig. 6 Experimental results of deformations of the actuator

The material properties that are applied to the Linear Elastic isotropic nitrile actuator are elastic modulus of $3000 \mathrm{MPa}$, Poisson's ratio 0.394 , mass density $1110 \mathrm{~kg} / \mathrm{m}^{3}$, Shear Modulus of $3.189 \times 10^{8} \mathrm{~N} / \mathrm{m}^{2}$.

\section{PROTOTYPE OF SIX LEGGED MICRO WALKING ROBOT}

The micro walking robot consists of six FMA legs each $12 \mathrm{~mm}$ in diameter and $100 \mathrm{~mm}$ in length. The overall dimensions of micro robot are $150 \mathrm{~mm}$ in length, $120 \mathrm{~mm}$ wide and $130 \mathrm{~mm}$ height. Fig. 7 shows the Prototype of the six legged micro walking robot.

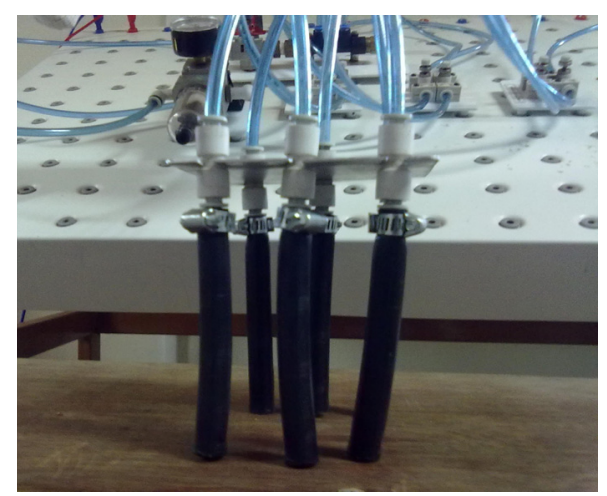

Fig. 7 Prototype of the six legged micro walking robot

\subsection{MOTION ALGORITM FOR THE ROBOT}

As shown in Fig. 8, FMA's 2 and 5 are used for the structural support of the robot when the other FMA's are under the pressure. FMA's 1 and 4, FMA's 3 and 6 are in synchronous action and starts actuation alternately.

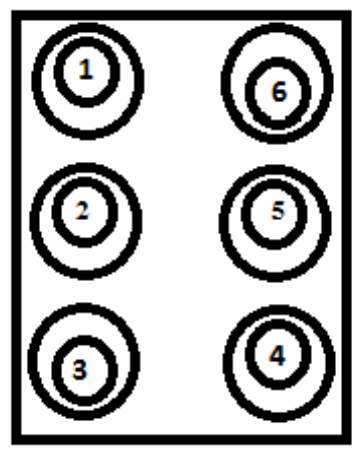

(a)

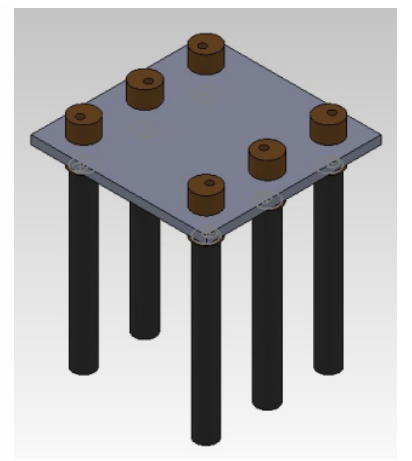

(b)
Fig. 8 (a) Arrangement for six FMA's for on off pressure control. (b) Solidworks model for FMA's.

Depending on the Flex sensor value read by the DAQ, the switching of the FMA's takes place. As the FMA has one degree of freedom the robot can move in one direction. To accomplish Omni-directional motion the future work includes the rotation of FMA by the micro motors attached to the FMA through gears.

\section{ELECTRO-PNEUMATIC CONTROL SYSTEM}

One of the important parts in micro robot is the control system which determines how the motion of the robot should take place. It happens by controlling each of the six legs individually restricting them to a particular deflection by following the motion mechanism which makes the robot to move in desired direction. To accomplish the motion by the robot, each FMA should follow the same cycle of operation as shown in Fig. 9. If each leg performs this walking cycle according to the control algorithm, motion can be achieved. FMA will be repeating this particular cycle during the motion of the robot.

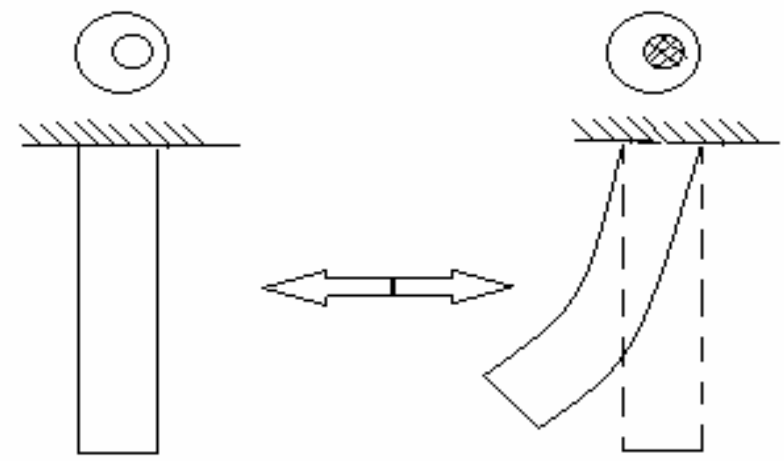

Fig. 9 FMA leg walking cycle. 


\subsection{CONTROL SYSTEM FOR SINGLE FMA}

When pressure is applied, FMA bends continuously as long as the pressure is increased but there is no control over the bending of the FMA for a desired angle of deflection. This can be accomplished by detecting the deflection using a sensor.

To measure such deflections or bending, two types of sensors available in the market are Strain gauges and Flex resistors. We have used both sensors and found that the Flex resistor is more suitable for FMA. Flex resistors are very easy to mount on any platform and the variation with respect to bending is also high compared to strain gauges.

Fig. 10 shows the hierarchy of the control system of a single FMA.

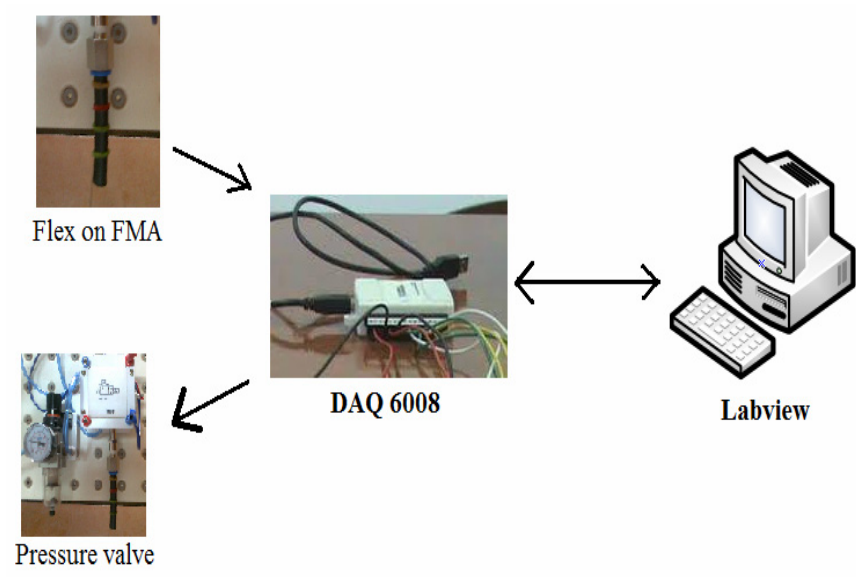

Fig. 10 Hierarchy of the system

The property of flex resistor is that the resistance increases if there is any bending. This happens because of the carbon resistive elements inside the flex sensor. By following the walking cycle of FMA, program is written in Labview software such that each FMA follows the control algorithm as shown in the Fig. 11.

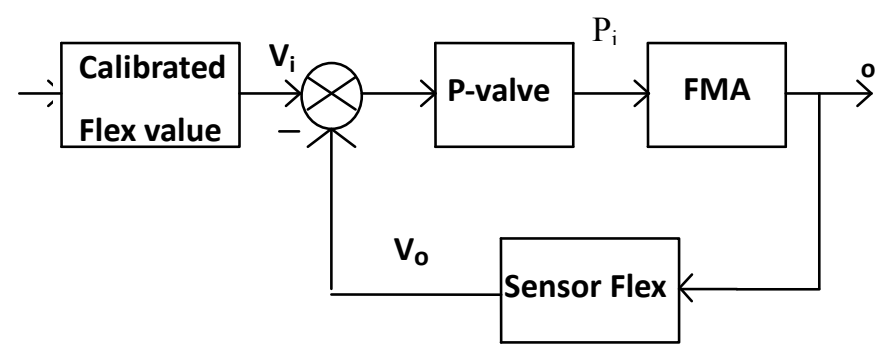

Fig. 11 Block diagram of the control system
The calibrated Flex value is the voltage $\left(\mathrm{V}_{\mathrm{i}}\right)$ corresponding to the desired value of the deflection ( $i$ ) of the FMA. When the pressure is passed through the FMA, it starts bending and the sensor flex converts this bending angle ( $\mathrm{o})$ to voltage $\left(\mathrm{V}_{\mathrm{o}}\right)$. This voltage is compared with the calibrated value $\left(\mathrm{V}_{\mathrm{i}}\right)$ and the pressure valve will be closed when both the values become equal. Fig. 12 shows the experimental setup of the single FMA which consists of pressure gauge, electronic pressure valve and the multimeter. The pull up resistance in the voltage divider circuit is the flex resistor and the voltage is given as input to the DAQ 6008 [10].

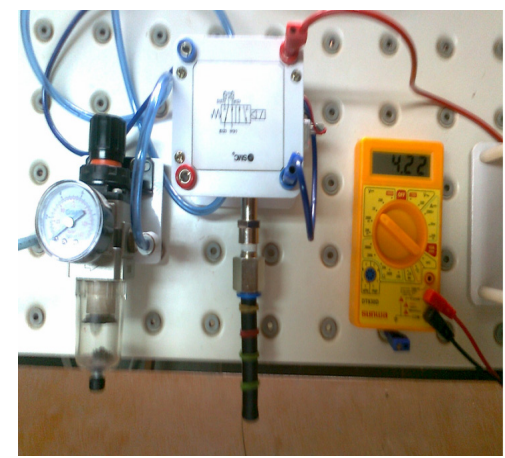

(a)

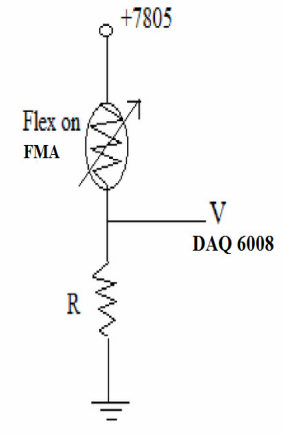

(b)
Fig. 12(a) Experimental setup of the single FMA. (b) Voltage divider circuit.

The initial resistance of the flex resistor (Spectra Symbol) is $7.6 \mathrm{k} \Omega$. It bends according to the pressure applied and results in the voltage change which is input to the DAQ. Table I shows various parameters of FMA with respect to pressure.

Table I

Parameters of FMA with respect to pressure

\begin{tabular}{|c|c|c|c|}
\hline $\begin{array}{c}\text { Pressure } \\
(\mathrm{MPa})\end{array}$ & $\begin{array}{c}\text { Deflection } \\
\text { (degrees) }\end{array}$ & $\begin{array}{c}\text { Radius of } \\
\text { curvature(mm) }\end{array}$ & $\begin{array}{c}\text { Voltage(input } \\
\text { to DAQ)(V) }\end{array}$ \\
\hline 0.2 & 30.1 & 158 & 4.21 \\
\hline 0.24 & 39 & 126 & 4.19 \\
\hline 0.28 & 42 & 113.3 & 4.17 \\
\hline 0.32 & 45 & 103 & 4.14 \\
\hline 0.33 & 54 & 92.2 & 4.10 \\
\hline 0.38 & 58.5 & 75.6 & 3.99 \\
\hline
\end{tabular}




\section{CONCLUSION}

A micro walking robot using FMAs as robot legs has been designed and fabricated. Since the walking robot has frictionless mechanism and no conventional links it can walk with gentle and animal like moments. A prototype for six legged mammalian type robot with electro-pneumatic control system has been developed for the implementation of motion mechanism. This walking robot made of single chamber FMA as compared to the earlier research has relatively less weight and less complicated control system and the robot can be easily miniaturized. It is clean without dust and no lubrication due to absence of sliding parts and thus can be used in clean environment.

\section{ACKNOWLEDGMENT}

The authors would like to thank N.S. Bhat, DGM, R and D, M/s ELGI Ultra Industries Ltd., Coimbatore for his assistance in mold manufacturing. We would also like to thank Mr. Kanakaraj V of Amrita Vishwa Vidyapeetham for his assistance in conducting the experiments.

\section{REFERENCES}

[1] T.Fukuda et al., "Rubber gas actuator driven by hydrogen storage alloy for In-pipe inspection mobile robot with flexible structure", in Proc. IEEE Int. Conf. Robotics and Automation, pp. 1847-1852, 1989.

[2] K.Suzumori, T. Maeda, H. Watanabe and T.Hisada.," Fiberless flexible microactuator," designed by FiniteElement method, IEEE/ASME Transactions on Mechatronics, vol.2 ,no. 4, pp.281-286, Dec.1997

[3] K.Suzumori, Micro-Walking Robot Driven by Flexible Microactuator, Journal of Robotics and Mechatronics Vol.5 No.6,1993, pp.537-541.

[4] K.Suzumori, et al., In-pipe Inspection Robot for 2B Pipelines,Proc. $8^{\text {th }}$ Conf. Robotics Society of Japan, (1990), 203.

[5] K.Suzumori, et, al., "Applying Flexible Micro actuator to Pipeline Inspection Robot", Transactions of the IMACS $\backslash$ SICE International Symposium on Robotics,
Mechatronics and Manufacturing Systems Kobe Japan, 1620 September, 1992.

[6] K. Suzumori, et al., "Applying A Flexible Microactuator to Robotic Mechanisms," IEEE Control Systems, vol.12, no.1, pp. 21-27, Feb1992.

[7] Ganesha Udupa and R. Krishna Murthy., "A new Flexing Technique for Soft Gripper Design," Proc. 16th All India Manufacturing Technology Design and Research Conference, Bangalore, pp.353-358. Dec 1994.

[8] Ganesha Udupa, et al, "Robotic Gripper Driven by Flexible Micro actuator Based on an Innovative Technique", IEEE Workshop on Advanced Robotics and its Social Impacts, KIST, Seoul, Korea, Oct 2010.

[9] S.P Timoshenko, Strength of Materials, Part I \& II, CBS Publishers, New Delhi, 2004.

[10] Adel S.Sedra and Kenneth C.Smith, "Microelectronic Circuits ", fifth edition, Oxford University Press, 2004. 